\title{
Resultados do tratamento cirúrgico da síndrome do túnel do tarso posterior idiopática
}

\author{
Leandro Pretto Flores ${ }^{1}$ \\ Unidade de Neurocirurgia do Hospital de Base do Distrito Federal, Brasília, DF, Brasil
}

\begin{abstract}
RESUMO
Objetivo: Demonstrar o resultado obtido com o tratamento cirúrgico da síndrome do túnel do tarso, para os casos ditos idiopáticos. Método: Análise retrospectiva de nove casos operados entre 2002 e 2005 , em pacientes nos quais se excluiu tumores, trauma ou doenças sistêmicas como etiologia. Resultado: Dor e parestesia na região plantar, de predominância noturna, associado a sinal de Tinel positivo no tornozelo foram as apresentações clínicas mais comuns. Os estudos eletrofisiológicos demonstraram aumento da latência sensitiva nos nervos plantares em todos os casos. Houve melhora dos sintomas em todos os pacientes submetidos à secção cirúrgica do retináculo dos flexores no tornozelo. A complicação mais observada foi deiscência da ferida operatória, identificada em seis pacientes. Conclusão: $A$ cirurgia é um método eficiente para o tratamento da síndrome do túnel do tarso nos casos idiopáticos, sendo o conhecimento anatômico fundamental para a realização da completa liberação de todos os potenciais pontos de compressão das estruturas nervosas da região.
\end{abstract}

\section{PALAVRAS-CHAVE}

Síndrome do túnel do tarso. Cirurgia.

\section{ABSTRACT}

Results of the surgical treatment of the idiopathic posterior tarsal tunnel syndrome

Objective: To demonstrate the results of the surgical treatment for idiopathic posterior tarsal tunnel syndrome. Method: A retrospective analysis of nine cases submitted to tarsal tunnel surgical decompression, between 2002 to 2005, was done. There was no case of space-occupying lesions or systemic diseases as etiological factors. Results: The most frequent clinical presentation was nocturnal pain and paraesthesia in the sole of the affected foot, associated to positive Tinel sign at the ankle. Electrophysiological studies demonstrated delay of the the sensitive conduction in plantar nerves in all cases. There was improvement of the symptoms in all of the patients submitted to surgical section of the flexor retinaculum. There were six cases of operative wound dehiscence - the most frequent operative complication. Conclusion: Surgery is an effective method for the treatment of idiopathic tarsal tunnel syndrome. A thorough anatomical knowledge is required to perform a complete liberation of all potential points of nerve compression in this particular region.

\section{KEY WORDS}

Tarsal tunnel syndrome. Surgery.

\section{Introdução}

A síndrome do túnel do tarso (STT) posterior é a denominação utilizada para a neuropatia compressiva do nervo tibial na região do tornozelo ${ }^{1}$. As manifestações clínicas são semelhantes àquelas observadas na síndrome do túnel do carpo, porém, relacionadas ao pé: queixas de dor e parestesias ocorrendo na região plantar e dedos, de predominância noturna. Recebe o nome de túnel do tarso posterior para diferenciar da síndrome do túnel do tarso anterior, esta última definida como a compressão do nervo fibular profundo pelo retináculo dos extensores. Pode ser de etiologia primária (idiopática) ou secundária a uma série de patologias da região do tornozelo, como tumores (lipomas, schwanomas, cistos ou gânglios), traumas, variantes anatômicas (ossos ou músculos acessórios), doenças vasculares (varicosidades), doenças endocrinológicas (hipotiroidismo) ou infecciosas (hanseníase) ${ }^{16}$. O tratamento recomendado inicialmente é o conservador, orientando-se repouso, 
fisioterapia e uso de analgésicos orais. Nos casos em que não há resposta clínica a essas manobras, recomenda-se o tratamento cirúrgico ${ }^{18}$.

O objetivo deste trabalho é demonstrar os resultados obtidos com o tratamento cirúrgico desta entidade clínica, com a identificação dos potenciais benefícios e riscos do procedimento, para os casos em que são excluídos fatores secundários como causa dos sintomas.

\section{Anatomia cirúrgica}

O nervo tibial - o maior ramo da divisão do nervo ciático - após cruzar a fossa poplítea adota um trajeto descendente pela perna, estando localizado profundamente ao músculo solear. Ao aproximar-se do tornozelo, torna-se mais superficial - sendo recoberto apenas pela fáscia profunda da perna - para então penetrar na região conhecida como túnel do tarso9 .

O túnel do tarso tem seu teto composto pelo retináculo dos flexores - um espessamento da fáscia profunda da perna - e o assoalho composto pelos ossos talus e calcâneo. O retináculo dos flexores (também denominado ligamento laciniato) estende-se entre o maléolo medial e o calcâneo, recobrindo o feixe vásculo-nervoso. Esta estrutura anatômica forma um túnel por onde transitam o nervo tibial, a artéria e veias tibiais posteriores, além dos tendões dos músculos tibial posterior, flexor longo do hálux e flexor longo dos dedos. Distalmente ao retináculo, encontra-se a inserção fibrosa do músculo abdutor curto do hálux $(\mathrm{AbCH})$ e a inserção da fáscia $\operatorname{plantar}^{21}$ (Figura 1).

O nervo tibial divide-se em ramos plantar medial e plantar lateral, após dar origem ao ramo calcanear medial; esta divisão está, geralmente, localizada três centímetros após o nervo penetrar no túnel do tarso, no chamado eixo maléolo-calcâneo (Figura 2). Desse

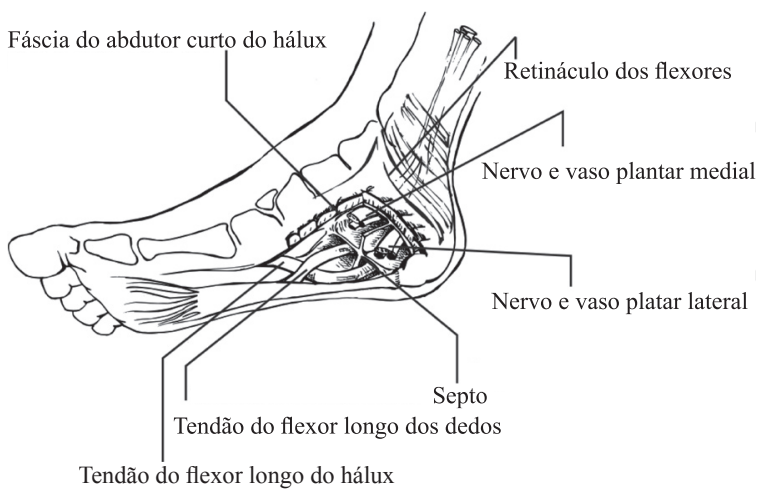

Figura 1

Anatomia regional do túnel do tarso. O desenho detalha as estruturas anatômicas identificadas após a ressecção do músculo abdutor curto do hálux.

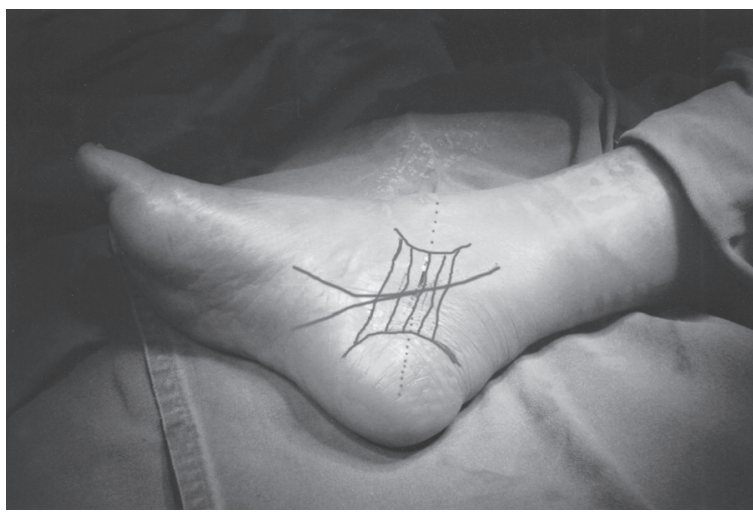

Figura 2

Anatomia de superfície do túnel do tarso: as duas marcas semicirculares correspondem ao maléolo medial (superior) e calcâneo (inferior); as linhas paralelas correspondem ao retináculo dos flexores; a linha mais marcada demonstra o curso do nervo tibial posterior e sua divisão em ramos plantar lateral e medial; a linha tracejada marca o eixo maléolo-calcâneo.

ponto, esses ramos seguem por túneis separados, que devem ser liberados individualmente para obter a completa descompressão cirúrgica. O nervo plantar medial segue um túnel cujo teto é composto pela inserção fibrosa do músculo AbCH e o nervo plantar lateral, outro túnel cujo teto é formado pela fáscia plantar, ambos são separados por um septo fibroso que se origina do periósteo do calcâneo e do tendão do músculo flexor longo do hálux, inserindo-se na transição entre a fáscia plantar e o músculo $\mathrm{AbCH}^{4}$ (Figura 3).

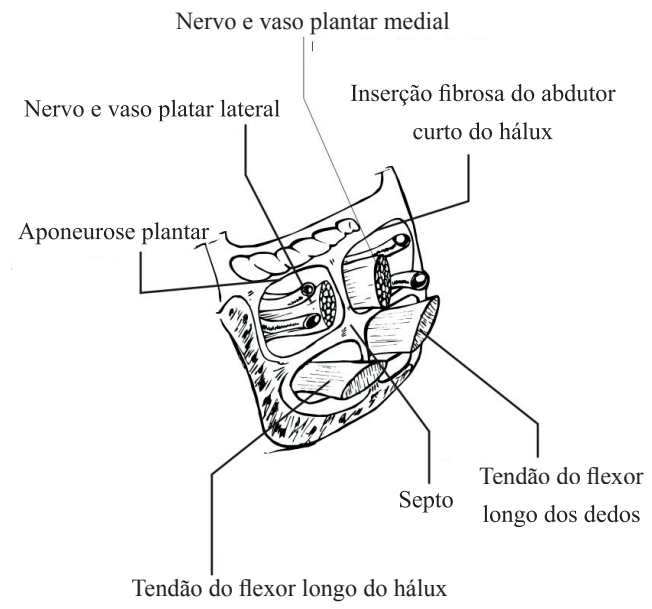

Figura 3

Esquema demonstrando a anatomia da porção do túnel do tarso distal ao retináculo dos flexores: observar que os nervos plantar lateral e plantar medial seguem por túneis separados, o mesmo ocorrendo com os tendões dos músculos flexor longo dos dedos eflexor longo do hálux. 
O ramo calcanear medial determina a sensibilidade da pele da região do calcanhar. Tem origem muito variada, podendo emergir do nervo tibial posterior ou de uma de suas divisões ${ }^{10}$. Pode também se apresentar como mais de um ramo: Dellon e cols. ${ }^{3}$, dissecando 85 tornozelos, identificaram este nervo como sendo tronco único em $37 \%$ dos pés, como dois ramos em $41 \%$, três ramos em $19 \%$ e como quatro em $3 \%$. Nesta série, o nervo originava-se mais freqüentemente do ramo plantar medial $(46 \%)^{3}$. O cirurgião deve estar atento a esse fato, pois lesão do(s) ramo(s) calcâneo pode resultar, além de hipoestesia no calcanhar, na formação de neuroma doloroso ${ }^{11}$, com conseqüente dor na ferida operatória.

\section{Métodos}

Foi realizado um estudo retrospectivo dos pacientes operados, pelo autor, para tratamento da síndrome do túnel do tarso posterior, no período de 2002 a 2005. Só foram incluídos os casos nos quais não foram identificados fatores secundários como etiologia do problema.

Os pacientes cujos sintomas sugeriam compressão do nervo tibial na região do tornozelo foram submetidos à eletroneuromiografia do membro acometido, para confirmação do diagnóstico. Todos os casos foram, inicialmente, tratados de maneira conservadora, com recomendação de repouso, fisioterapia ou uso de analgésicos orais. Em caso de falha dessa conduta, indicou-se o tratamento cirúrgico.

\section{Técnica cirúrgica}

Todas as cirurgias foram realizadas sob anestesia peridural, com paciente em decúbito dorsal. Não se usou garroteamento do membro inferior em nenhum caso. O procedimento foi realizado com auxílio de lupa cirúrgica, com magnificação de três vezes. É feita uma incisão curvilínea na face medial do tornozelo, localizada a dois centímetros do maléolo medial, iniciando quatro centímetros acima deste e estendendo-se até a região ínfero-medial do pé, no limite da região plantar. Após a abertura da fáscia profunda da perna, identificase o feixe neurovascular (nervo tibial e artéria e veias tibiais posteriores) na região proximal ao retináculo dos flexores. Isola-se o nervo e, tendo este sempre sob visibilização direta, procede-se completa secção do retináculo. Após isso, realiza-se a cuidadosa dissecção dos ramos plantar medial e lateral. Para a liberação do plantar medial, secciona-se a fáscia de inserção músculo $\mathrm{AbCH}$ e, para a descompressão do nervo plantar late- ral, procede-se à secção da porção proximal da fáscia plantar (Figura 4). Em geral, algumas veias de pequeno e médio calibres são identificadas cruzando sobre estes nervos, elas devem ser sempre coaguladas e secionadas, com o objetivo de evitar pontos de compressão neurovascular. O septo fibroso que se interpõe entre os nervos não foi ressecado como rotina, assim como não foi realizada epineurólise em nenhum paciente. A síntese da ferida foi feita com suturas subcutâneas usando-se fios absorvíveis, sendo a pele suturada com pontos separados usando-se fio de náilon 4.0.

Os pacientes, em geral, receberam alta no dia seguinte à cirurgia. Recomendava-se manter elevação do membro inferior operado por 72 horas e evitar apoiar o pé no chão por uma semana. Os pontos de sutura cutânea foram retirados no 14ํㅜㄹ dia de pós-operatório.

\section{Resultados}

No período considerado para o estudo, foram operados dez pacientes com sintomas compatíveis com síndrome do túnel do tarso posterior. Um paciente apresentava lipoma como etiologia, sendo excluído do trabalho. No total, nove casos compõem o grupo de estudo, nos quais não havia fator causal identificável; sete pacientes eram do sexo feminino e dois, do masculino. A idade dos pacientes variou entre 33 e 52 anos, com média de 44 anos. Quanto ao tempo de evolução da doença, observou-se um caso com sintomas há menos

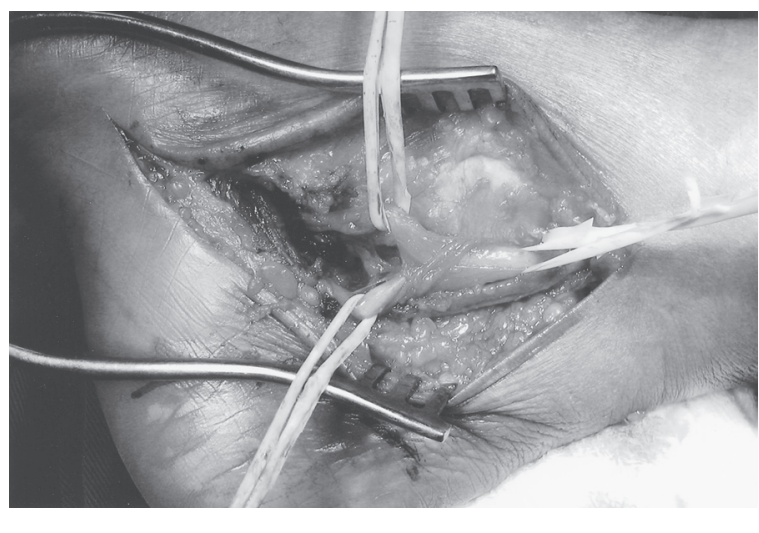

Figura 4

Dissecção cirúrgica final, após a completa secção do retináculo dos flexores, inserção fibrosa do músculo $\mathrm{AbCH}$ e fáscia plantar, liberação dos nervos tibial posterior, plantar medial (superior na figura) e plantar lateral (inferior na figura). 
de seis meses; cinco entre seis meses e um ano; e três com sintomatologia há mais de um ano.

A tabela 1 resume as principais manifestações clínicas neste grupo. Dor acompanhada de parestesias na região plantar e dedos, de predominância noturna, foi a principal queixa identificada. Esse padrão de história clínica normalmente vinha associado com positividade do sinal de Tinel sobre a região medial do tornozelo (77\%). Também se observou que a manobra de compressão do nervo tibial foi altamente sensível para o diagnóstico clínico da doença. Esta última consiste em comprimir a região do túnel do tarso por dois minutos, sendo considerada positiva, caso provoque parestesias nas pontas dos dedos do pé durante a manobra.

Em dois pacientes as manifestações clínicas eram bilaterais. Para esta situação, optou-se, inicialmente, pela descompressão do lado que o paciente referia ser mais sintomático. Nos dois casos, observou-se melhora dos sintomas no pé oposto após à cirurgia, não necessitando nova intervenção.

Os estudos eletrofisiológicos foram realizados em todos pacientes, sendo seus resultados positivos considerados obrigatórios para a confirmação diagnóstica. O aumento da latência da condução sensitiva nos nervos plantar medial e lateral foi a alteração mais confiável para o diagnóstico, sendo observada em todos os casos. Desnervação muscular, especialmente em músculos abdutor curto do hálux ou abdutor do dedo mínimo, foi observada somente em cinco casos. Estes estudos foram repetidos dois meses após a cirurgia, tendo sido observada redução da latência sensitiva em oito pacientes e nenhuma alteração em um caso.

Cinco pacientes com dor lombar associada foram investigados também com RM da coluna, para diagnóstico diferencial de radiculopatia lombo-sacra. Esta se mostrou normal em dois pacientes, mas alterada em três: um paciente apresentava hérnia de disco mediana de base larga no espaço L5-S1; outro, estenose forami-

Tabela 1. Manifestações clínicas observadas em nove pacientes com diagnóstico de síndrome do túnel do tarso posterior idiopática.

\begin{tabular}{ll}
\hline Sintomatogia & n \\
Dor na sola do pé & 9 \\
Parestesias em sola do pé e dedos & 9 \\
Piora noturna da dor & 9 \\
Manobra de compressão positiva & 8 \\
Sinal de Tinel positivo & 7 \\
Irradiação dor para panturrilha & 6 \\
Piora da dor ao caminhar & 4 \\
Hipoestesia em dedos & 3 \\
Perda de força nos dedos* & 3 \\
*Notada pelo paciente &
\end{tabular}

nal L5-S1 bilateral; e o terceiro, discoartrose (Modic tipo II) em L5-S1.

Observou-se que nenhum paciente apresentou melhora dos sintomas apenas com o tratamento conservador. Em geral, o repouso somente aliviava transitoriamente a dor, mas não se mostrou como opção definitiva para o problema. Fisioterapia e analgésicos orais (antiinflamatórios não-hormonais) não apresentaram resultados sensíveis aos pacientes. Assim, todo o grupo foi submetido à cirurgia. Durante o ato cirúrgico, observou-se a presença de veias cruzando o trajeto de algum dos nervos em seis pacientes. A melhora dos sintomas ocorreu em todos os pacientes após o procedimento: a resolução da dor e da parestesia freqüentemente foi observada já na primeira semana após a cirurgia, enquanto a hipoestesia de dedos (observada em três casos) tendeu a mostrar melhora somente após dois a três meses. O tempo de seguimento desses pacientes variou de quatro meses a dois anos.

As complicações observadas foram: cinco casos de infecção de ferida operatória, seis casos de deiscência de ferida e dois casos de hipoestesia de calcâneo. As infecções foram limitadas aos tecidos superficiais, observando-se eritema e edema da ferida e, em geral, ocorreram após o sétimo dia pós-operatório. Todas foram tratadas com antibióticos orais, com resolução. As deiscências surgiam após a retirada dos pontos de sutura, ocorrendo, na maioria das vezes, na porção proximal da ferida operatória. Foram tratadas com curativos locais para síntese por segunda intenção. Os dois pacientes que apresentaram hipoestesia de calcâneo recuperaram o déficit neurológico em até dois meses.

\section{Discussão}

A síndrome do túnel do tarso posterior é uma neuropatia compressiva do nervo tibial e/ou dos seus ramos terminais: plantar lateral e medial ${ }^{1}$. Quando idiopática, em geral duas estruturas estão envolvidas no mecanismo de compressão: o retináculo dos flexores, que pode comprimir o nervo tibial ou seus ramos na região do túnel do tarso e/ou a inserção fibrosa tanto do músculo $\mathrm{AbCH}$ quanto da fáscia plantar, que podem causar compressão sobre os nervos plantar medial ou lateral, isoladamente. Essas possibilidades são indistinguíveis clinica e/ou eletrofisiologicamente, portanto, a cirurgia a ser realizada nesses casos deve sempre incluir a completa descompressão de todos estes potenciais pontos de compressão ${ }^{17}$.

Anatomicamente, a região do túnel do tarso em muitos aspectos se parece com o túnel do carpo: um nervo que penetra em uma extremidade através de um 
túnel ósteo-fibroso. Porém, as analogias terminam por aí: os estudos de Dellon e Mackinnon ${ }^{5}$ demonstraram que o canal do carpo é análogo, na verdade, ao túnel de trânsito do nervo plantar medial, sendo a inserção fibrosa do músculo $\mathrm{AbCH}$ a estrutura correspondente, no punho, ao ligamento transverso do carpo. O túnel para o nervo plantar lateral seria análogo ao canal de Guyon, sendo a fáscia plantar correspondente ao ligamento volar do carpo. No punho, o hamato separa o túnel do carpo do canal de Guyon; na região do túnel do tarso, essa função é exercida pelo septo que se interpõe entre os dois nervos. O retináculo dos flexores do tornozelo corresponderia, no antebraço, ao espessamento progressivo da fáscia antebraquial, que a seguir se transforma no ligamento transverso do carpo ${ }^{5}$. $\mathrm{Na}$ síndrome do túnel do carpo, apenas a secção desta fáscia não é o suficiente para o alívio dos sintomas do paciente, sendo o mesmo raciocínio utilizado para a cirurgia dos casos de síndrome do túnel do tarso.

O quadro clínico dos pacientes desta série não difere de outros estudos a respeito do mesmo assunto, pois parece bastante típico ${ }^{14}$. Porém, não é incomum observar-se a associação entre túnel do tarso e doença degenerativa da coluna lombar. Essa associação pode dificultar a identificação diagnóstica da STT, confundindo os sintomas como parte de uma radiculopatia lombo-sacra ${ }^{2}$, especialmente nos pacientes que também se queixam de dor lombar. Nesta série, observou-se que alguns casos já vinham de outros serviços com diagnóstico de doença da coluna vertebral firmado, estando já em tratamento - obviamente sem alívio dos sintomas. Os estudos de imagem da coluna demonstraram alterações em três pacientes. Observou-se, também, a irradiação da dor para a panturrilha em seis casos. Associado a esses fatos, é importante lembrar que os estudos eletrofisiológicos na síndrome do túnel do tarso são, muitas vezes, de difícil interpretação, podendo um examinador inexperiente não reconhecer ou não identificar as alterações características da doença ${ }^{6}$. Portanto, o diagnóstico dessa síndrome é teoricamente fácil - considerando-se a apresentação clínica clássica - mas, os elementos acima citados podem confundir a conclusão diagnóstica, conseqüentemente atrasando o tratamento definitivo.

No presente estudo, foi possível observar alguns sinais e sintomas que devem sempre alertar o cirurgião quanto ao diagnóstico de STT: dor de predominância noturna; parestesia na região plantar, sinal de Tinel positivo no tornozelo e manobra de compressão positiva. A presença dessas manifestações semiológicas facilita o diagnóstico diferencial com radiculopatia de S1.

A eletroneuromiografia é o exame complementar mais importante para o diagnóstico. Na presente série, os estudos de condução se mostraram mais confiáveis do que a eletromiografia. Isto porque a desnervação dos músculos estudados nem sempre foi observada, ao passo que o retardo na condução sensitiva foi o dado que permitiu o diagnóstico. Também, é necessário ponderar que alguns indivíduos normais apresentam desnervação crônica da musculatura intrínseca do pé - provocada por traumas repetidos decorrentes da prática de alguns esportes ou ao uso de calçados apertados - condições estas que podem determinar resultados falso-positivos nos estudos eletromiográficos ${ }^{6}$.

Analisando 59 pacientes com diagnóstico de STT, dos quais 23 eram idiopáticos, Mondelli e cols. ${ }^{15}$ observaram retardo na condução sensitiva em $77 \%$ dos casos, retardo na condução motora em $55 \%$ e desnervação em $45 \%$. Na presente série, os estudos eletrofisiológicos foram também realizados no período pós-operatório e demonstraram que a melhora clínica, após o tratamento cirúrgico, em geral é acompanhada de melhora eletrofisiológica - observando-se a redução da latência sensitiva em oito pacientes. Ward e Porter ${ }^{20}$, em 1998, já haviam identificado que a melhora dos estudos de condução normalmente acompanha a melhora clínica.

Os casos de STT ditos idiopáticos são aqueles nos quais não se identifica um fator secundário para o desenvolvimento dos sintomas. Isto significa a exclusão de doenças sistêmicas - sejam infecciosa, endocrinológica ou reumatológica - como fator causal e, durante o ato operatório, não deve ser observada nenhuma lesão ocupando espaço no túnel do tarso. Por outro lado, nos procedimentos realizados nesta série, identificou-se com freqüência a presença de muitas veias de médio calibre cruzando o trajeto dos nervos envolvidos. Pode-se argumentar, dessa forma, em favor de uma etiologia neurovascular para a compressão do nervo tibial posterior ou de seus ramos. Gould e Álvares ${ }^{8}$ já relataram um caso de síndrome do túnel do tarso bilateral causada por varicosidades no tornozelo. Porém, ainda não foram desenvolvidos estudos anatômicos comparando as relações veno-nervosas em tornozelos ditos sadios com aqueles de pacientes com STT. Kohno e cols. ${ }^{13}$, em 2000, sugeriram a existência da compressão neurovascular na síndrome do túnel do tarso idiopática e, devido a isso, reportaram uma técnica de interposição de fragmento de tecido gorduroso entre os nervos e as veias. Na presente série, considerando esta possibilidade, o autor preferiu a coagulação e secção das veias que cruzavam o trajeto nervoso.

O estudo de Flaningan e cols. ${ }^{7}$, a respeito da irrigação dos nervos no túnel do tarso, demonstrou que estes têm seu suprimento vascular proveniente diretamente das artérias adjacentes (ramos diretos da artéria tibial posterior) e que estas artérias supridoras podem ser, teoricamente, comprimidas pelo retináculo dos flexores. Assim, pode-se também considerar a compressão arterial, com conseqüente isquemia intraneural, como possível fator etiológico para casos ditos idiopáticos. 
Finalmente, em analogia com a síndrome do túnel do carpo, poder-se-ia considerar o aumento da pressão compartimental devido à tenosinovite crônica inespecífica como a causa da STT idiopática. No entanto, ampla revisão bibliográfica sobre o tema não encontrou qualquer estudo provando que isso realmente ocorra. Além disso, não se identifica qualquer atividade de risco (profissional) relacionada ao desenvolvimento da STT, diferentemente da síndrome do túnel do carpo. Independente do fator etiológico, a secção completa do retináculo e dos outros potenciais pontos de compressão nervosa permite a resolução dos sintomas. Em alguns pacientes foi identificada a presença de veias sobre os nervos, em outros não. Portanto, o fator mais importante parece ser os elementos fibrosos e, por isso, todos os esforços devem ser voltados para sua total secção ${ }^{12}$.

As complicações observadas estão mais diretamente relacionadas à região operada. A região do tornozelo - por sofrer de pobre irrigação, sustentar o peso do corpo, apresentar grande grau de mobilidade, dentre outros motivos - apresenta alto índice de infecção de ferida e deiscência de sutura, independentemente do tipo de cirurgia realizada ${ }^{19}$. Os casos de hipoestesia de calcanhar mostraram-se reversíveis em alguns meses, provando ser de origem neuropráxica, e não por lesão direta do ramo calcanear medial. Isso provavelmente ocorreu devido à técnica cirúrgica, com eventual tração dos nervos durante a dissecção, provocando estiramento do referido ramo.

\section{Conclusão}

A cirurgia é eficiente para o tratamento da síndrome do túnel do tarso posterior idiopática, desde que realizada com objetivo de liberação de todos os pontos potenciais de compressão dos nervos - retináculo dos flexores, inserção fibrosa do músculo $\mathrm{AbCH}$ e da fáscia plantar. Recomenda-se também a secção de veias que, ocasionalmente, cruzam sobre as estruturas nervosas, para evitar manutenção de pontos de compressão neurovascular. O conhecimento anatômico da região é fundamental para a completa descompressão de todos os elementos nervosos envolvidos.

\section{Agradecimento}

O autor agradece ao Sr. Eduardo de Sousa Ribeiro Dias pela colaboração realizando os desenhos de anatomia apresentados neste artigo.

\section{Referências}

1. AGUIAR PHP, TELLA Jr OI, TEDESCO-MARCHESE A: Síndrome do Túnel do Tarso Posterior. In Tatagiba M (ed): Nervos Periféricos - Diagnóstico e Tratamento Clínico e Cirúrgico. Rio de Janeiro, Revinter, 2003, pp 63-5.

2. BABA H, WADA M, ANNEN S, AZUCHI M, IMURA S, TOMITA K: The tarsal tunnel syndrome: evaluation of surgical results using multivariate analysis. Int Orthop 21:67-71, 1997.

3. DELLON AL, KIM J, SPAULDING CM: Variations in the origin of the medial calcaneal nerve. J Am Podiatr Med Assoc 92: 97-101, 2002.

4. DELLON AL, MACKINNON SE: Tibial nerve branching in the tarsal tunnel. Arch Neurol 41:645-6, 1984.

5. DELLON A, MACKINNON SE: Tarsal Tunnel Syndrome. In Dellon A, Mackinnon SE (eds): Surgery of the Peripheral Nerves. Sttutgart, Thieme Med. Publishers, 1988, pp 305-18.

6. FERREIRA AS: Nervo ciático e suas divisões fibular comum e tibial. In Ferreira AS (ed): Lesões Nervosas Periféricas. São Paulo, Ed. Santos, $2^{a}$ edição, 2001,181-94.

7. FIANINGAN DC, CASSELL M, SALTZMAN CL: Vascular supply of nerves in the tarsal tunnel. Foot Ankle Int 18: 28892, 1997.

8. GOULD N, ALVARES R: Bilateral tarsal tunnel syndrome caused by varicosities. Foot Ankle 3: 290-2, 1983.

9. GRAY H, GOSS CM: Anatomia. Rio de Janeiro, Ed. Guanabara Koogan, $2^{\mathrm{a}}$ Edição, 1988.

10. HORWITZ MT: Normal anatomy and variations of the peripheral nerves of the leg and foot. Arch Surg 36:636-46, 1938.

11. KIM J, DELLON AL: Neuromas of the calcaneal nerves. Foot Ankle Int 22:890-4, 2001.

12. KIM DH, RYU S, TIEL RL, KLINE DG: Surgical management and results of 135 tibial nerve lesions at the Lousiana State University Health Science Center. Neurosurgery 53:1114-24, 2003

13. KOHNO M, TAKANHASHI $\mathrm{H}$, SEGAWA $\mathrm{H}$, SANO $\mathrm{K}$ : Neurovascular decompression for idiopathic tarsal tunnel syndrome: technical note. J Neurol Neurosurg Psychiatry 69: 87-90, 2000.

14. LAU JT, DANIELS TR: Tarsal Tunnel Syndrome: a review of the literature. Foot Ankle Int 20:201-9, 1999.

15. MONDELLI M, GIANNINI F, REALE F: Clinical and eletrophysiological findings and follow-up in tarsal tunnel syndrome. Electroencephalogr Clin Eletrophysiol 109: 418-25, 1998.

16. MUMENTHALER M, SCHLIACK H: Lesions of individual nerves of the lower limb plexus and the lower extremities. In Mumenthaler M, Schliack H (eds.): Peripheral Nerve Lesions - Diagnosis and Therapy. Sttutgard, Thieme Med. Pub, 1991, pp 297-343.

17. RADIN EL: Tarsal Tunnel Syndrome. Clin Orthop 181:16770, 1983.

18. SETTANI FA, LEANDRO LM, ZULETA JA, NERI EA: Management of tarsal tunnel syndrome. Report of seven cases. Arq Neuropsiquiatr (S Paulo) 52: 530-4, 1994.

19. TAKAKURA Y, KITADA C, SUGIMOTO K, TANAKA Y, TAMAI S: Tarsal tunnel syndrome. Causes and results of operative treatment. J Bone Joint Surg $(\mathrm{Br})$ 73:125-8, 1991.

20. WARD PJ, PORTER ML: Tarsal tunnel syndrome: a study of the clinical and neurophysiological results of decompression. JR Coll Surg Edinb 43:35-6, 1998.

21. WOOD BM: Compressive neuropathies of the lower extremity. In Gelberman RH (ed): Operative Nerve Repair and Reconstruction. Philadelphia, J.B. Lippicott Co., Vol. 2, 1991, pp 1197-210. 
Original recebido em julho de 2005

Aceito para publicação em setembro de 2005

\section{Endereço para correspondência:}

Leandro Pretto Flores

SQN 208, Bloco F, Ap 604

70853-060 - Brasília, DF, Brasil

Email:leandroprettoflores@hotmail.com

\section{Comentários}

O autor apresenta a análise de uma série de nove pacientes com a síndrome do túnel do tarso posterior submetidos a tratamento cirúrgico, coletada em um curto período de tempo, sobre a qual gostaríamos de tecer alguns comentários:

- Conforme ressaltado no texto, a porção proximal da incisão deve ser realizada a alguma distância do maléolo medial. Tal preocupação tem por finalidade evitar a lesão de ramos terminais do nervo safeno.
- Na tentativa de anular todos os possíveis pontos de compressão, de forma diferente a preconizada pelo autor, seccionamos, de rotina, o septo fibroso posicionado entre os nervos plantares. Dessa forma, criamos um túnel amplo e único para a passagem dos dois nervos.

- A deiscência da ferida operatória é relativamente comum nesse tipo de cirurgia, conforme justificado no texto. Talvez, em nosso meio, esse problema seja potencializado pelo o número de pacientes com níveis nutricionais inadequados $\mathrm{e}$ que, por vezes, não compreendem a importância das recomendações médicas.

$\mathrm{O}$ autor deve ser parabenizado por seus resultados cirúrgicos e pela importante contribuição à literatura nacional relacionada ao tratamento cirúrgico de lesões de nervos periféricos.

Mário Siqueira Editor

Hospital das Clínicas da Faculdade de Medicina da Universidade de São Paulo Departamento de Nervos Periféricos da SBN 